\title{
Analyse de performances poétiques dans une joute de slam
}

\author{
JUDITH ÉMERY-BRUNEAU \\ Université du Québec en Outaouais, Canada \\ NYDIA PANDO \\ Universidad de Guadalajara, Mexique
}

\section{Résumé}

Dans cet article, nous présentons une analyse de performances poétiques observées dans le cadre d'une joute de slam. L'analyse de notre corpus, composé de 16 performances poétiques observées lors d'une finale régionale, nous a menées à identifier trois aspects prépondérants dans ces performances poétiques : 1) l'antinomie, l'anaphore et la litanie comme procédés récurrents pour créer l'empathie; 2) la purge bilatérale entre la catharsis des slameurs et celle des spectateurs; 3) l'humour comme gage d'engagement des spectateurs. Nous illustrons en quoi la performance poétique résulte de l'action qui s'opère dans la rencontre entre un texte original slamé avec la voix et le corps d'un slameur, et sa réception instantanée par des spectateurs qui y réagissent dans un moment - immédiat et éphémère -, un contexte et un espace déterminés.

\section{Mots-clés}

Performance poétique; slam; poésie; poésie orale; oralité.

\section{Analysis of poetic performances in a slam competition}

\section{Abstract}

In this article, we present an analysis of poetic performances observed in the context of a slam competition. The analysis of our corpus, composed of 16 poetic performances observed during a regional final, led us to identify three salient aspects in these type of performances: 1) anaphora, contradiction, and litany as recurring processes to create empathy; 2) bilateral purging between the catharsis of the slammers and their audience; and 3) humour as pledge of commitment of the audience. We illustrate how the poetic performance results from the action that occurs in the encounter between an original text delivered with the voice and the body of a slammer and the instantaneous reception by the audience reacting to the text in the immediate and ephemeral moment, a context and a defined space.

Keywords

Poetic performances; slam; poetry; oral poetry; orality. 


\section{Texte complet}

Le slam est un genre oral du poème écrit qui se réalise dans le cadre d'une joute entre plusieurs slameurs. Depuis son apparition à Chicago dans les années 1980 (Smith et Kraynak, 2009), ce genre, rapidement devenu un phénomène mondial ${ }^{1}$, a participé à une véritable révolution dans le milieu de la poésie (Gregory, 2008). En effet, le texte du slameur prend forme dans et par la voix et le corps : il fait image, laquelle provoque forcément des réactions chez les spectateurs qui voient et entendent la performance et même, jugent de son acuité et de son impétuosité. La joute de slam est donc composée de la rencontre tripartite de textes originaux, de performances de slameurs et de réactions des spectateurs (Émery-Bruneau et Yobé, 2015). Le slam a par conséquent démocratisé la poésie, permettant à toute personne intéressée de s'exprimer, d'entendre, de voir ou d'évaluer des slameurs sur scène. En d'autres mots, il s'agit d'une poésie circonstancielle et populaire prenant la forme d'un récitatif scandé (Zumthor, 1983 : 161).

Dans la première phase de notre programme de recherche, nous avions décrit le déroulement des joutes de slam et les caractéristiques textuelles du genre. Il s'agissait d'une étude sur la pratique sociale du slam au cours de laquelle nous analysions les discours, les signes et les codes entourant les joutes de slam (Émery-Bruneau et Yobé, 2015). Cette première phase présentait toutefois une analyse plutôt limitée des performances poétiques, dimension pourtant centrale dans la joute de slam. Or, si l'on considère que le slam est un genre de poésie orale, nous devons l'analyser, à l'instar de Zumthor (1983), en tant que discours circonstanciel foncièrement mouvant. Par ces « interventions circonstancielles » (Zumthor, 1987 : 160), le poète inscrit sa performance dans le présent d'un lieu et d'un auditoire. Partant, la performance poétique induit un rapport au temps spécifique, obligeant le slameur à s'éloigner d'un texte stabilisé à l'écrit, car contrairement à la poésie écrite, le slameur compose avec l'instantanéité, l'éphémère, la " mouvance " (Zumthor), la dialectique entre improvisation et réitérabilité. Nous nous sommes donc demandées comment, dans le cas des joutes de slam, les slameurs et les spectateurs embrassent-ils l'immédiateté et participent-ils à la création de performances poétiques. Qu'est-ce qui caractérise spécifiquement leurs performances poétiques entendues comme action orale-aurale (Zumthor, 1987 : 248)?

Dans cet $\operatorname{article}^{2}$, nous présentons une analyse illustrée des performances poétiques observées dans le cadre d'une joute de slam. L'analyse ${ }^{3}$ de notre corpus composé de 16 performances poétiques observées lors d'une finale régionale nous a menées à identifier trois aspects prépondérants dans ces performances : 1) l'antinomie, l'anaphore et la litanie comme procédés récurrents pour créer l'empathie; 2) la purge bilatérale entre la catharsis des slameurs et celle des spectateurs; 3) l'humour comme gage d'engagement des spectateurs. Nos analyses, appuyées d'exemples tirés de notre corpus, illustrent en quoi la performance poétique résulte de l'action qui s'opère dans la rencontre entre un texte original slamé avec la voix et le corps d'un slameur, et sa

\footnotetext{
${ }^{1}$ Par exemple, les premières compétitions officielles sont apparues en Angleterre en 1994, en Suisse romande (Genève) en 2002, à l'Ile Maurice en 2002, en France en 2004, à México en 2007, au Canada anglais (Ottawa) en 2004, au Québec (Montréal) en 2006, etc.

${ }^{2}$ Ce texte adopte l'orthographe rectifiée : http://www.orthographe-recommandee.info/

${ }^{3}$ Nous remercions l'Équipe de Recherche Littératie et Inclusion (ERLI) pour avoir financé le stage de recherche de Nydia Pando, ce qui lui a permis de participer à l'analyse des données pour cet article.
} 
réception instantanée par des spectateurs qui y réagissent dans un moment - immédiat et éphémère -, un contexte et un espace déterminés.

\section{Cadre théorique : joute de slam et performance poétique}

Selon Mark Kelly Smith, son fondateur, le slam est composé de cinq dimensions : un texte poétique, une performance d'un slameur, une compétition (joute), des interactions avec les spectateurs et une communauté de slameurs (Smith et Kraynak, 2009 : 14). Le slam est par conséquent associé à la performance poétique, comme l'a constaté l'ethnologue Gregory : "Performance poetry is commonly used within poetry communities to refer to work that is performed orally rather than presented on the page, and that allies itself more with the conventions of slam than with those of the "academic" poetry world. » (2008 : 204). En outre, bien que poésie de l'oral, ses liens avec l'écrit sont incontournables étant donné que le slam n'est pas un exercice d'improvisation comme peut l'être notamment le rap battle, très répandu dans le mouvement hip hop : «Structuré par l'oralité et par certains éléments formels tels que les rimes, le rythme, le débit, le flow, il favorise une mise en pratique équilibrée, de l'oral et de l'écrit, et permet de faire le lien avec les textes poétiques » (Brown, 2011 : 159). En ce qui concerne la « communauté » de cette pratique sociale de référence, il s'agit des personnes présentes aux joutes, soit des slameurs amateurs ou expérimentés, soit des spectateurs intéressés, curieux, passionnés par le slam (Émery-Bruneau et Yobé, 2015).

Pour définir la notion de performance telle qu'elle s'actualise dans une joute de slam, nous avons identifié cinq dimensions constitutives : un texte original du slameur écrit avant la réalisation de la performance; un temps déterminé; un espace déterminé; un slameur interprétant son texte original avec sa voix (et son silence), son corps (et ses gestes zéro) et les finalités de sa performance (ex. : faire rire, convoquer l'empathie, se purger, convaincre, etc.); et des réactions spontanées de spectateurs. Ces dimensions régissent les performances poétiques et nous pouvons en observer les interactions dans une joute de slam. Nous illustrons cette notion dans la figure 1 qui suit.

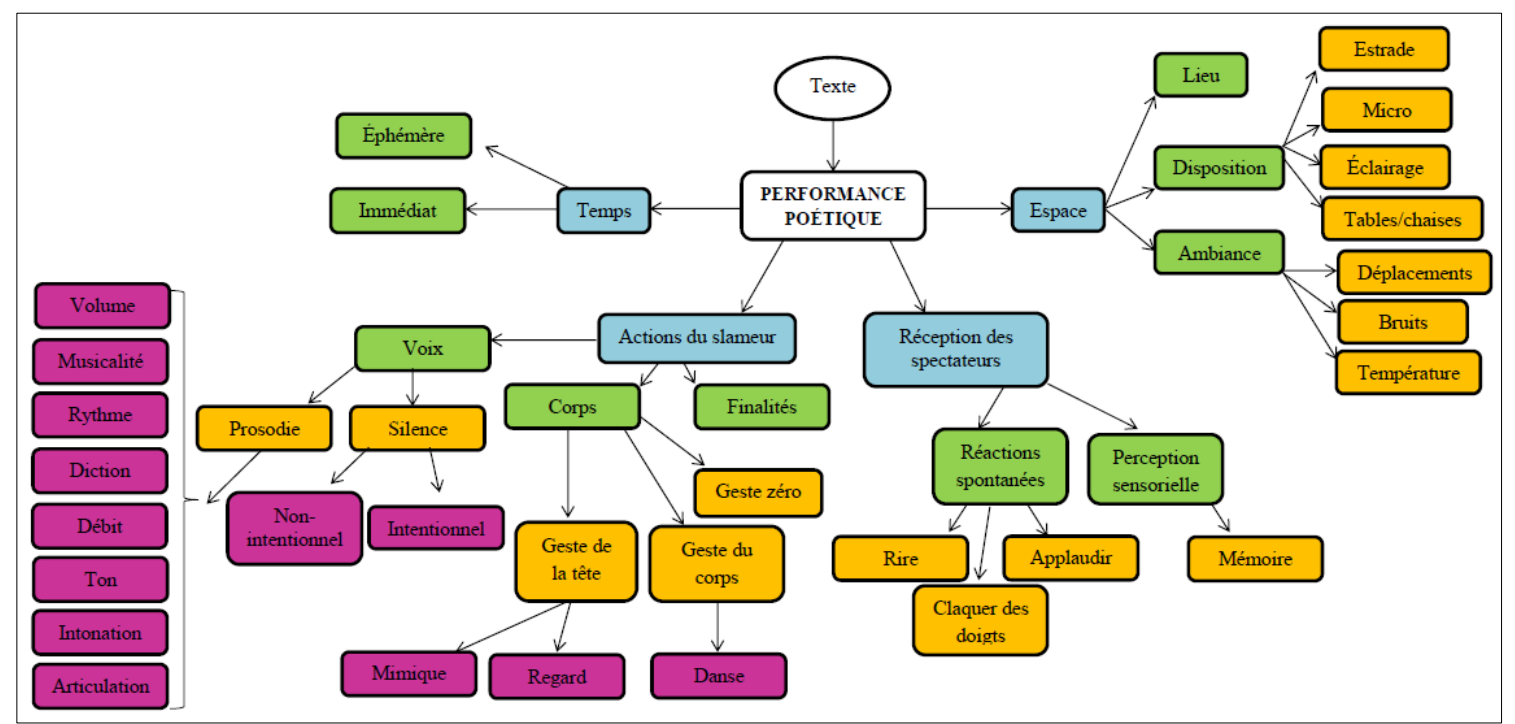

Figure 1. Carte conceptuelle de la notion de performance poétique 
Smith et Kraynak (2009) définissent la performance poétique de la scène slam ainsi : " The marriage of a text to the artful presentation of poetic words onstage to an audience that has permission to talk back and let the performer know whether he or she is communicating effectively » (2009: 5-6). Alors que le slam naissait à Chicago dans les années 1980, la notion de performance commençait à être théorisée en études littéraires, notamment par le médiéviste et spécialiste de la poésie orale Paul Zumthor, qui définissait la performance comme «l'action complexe par laquelle un message poétique est simultanément transmis et perçu, ici et maintenant» (Zumthor, $1983: 32$ ). Pour lui, il s'agit d'une «action orale-aurale complexe [...] [dans laquelle] se recoupent les deux axes de toute communication sociale : celui qui joint le locuteur à l'auteur; et celui sur quoi s'unissent situation et tradition » (Zumthor, 1987 : 248). La performance, comme celle actualisée dans le slam, est « destiné[e] à être dit[e], écouté[e], scandé[e], et non à être lu[e], sa forme sonore est primordiale » (Chetouani, 2011: 88). La notion de performance poétique renverrait donc à cette relation entre un texte et sa représentation artistique par un performeur, laquelle est située dans un temps et un espace où des spectateurs réagissent spontanément. Ces derniers constituent une variable essentielle pour qu'il y ait performance, car la performance ne peut échapper à l'interaction production-réception, le slameur s'adaptant aux spectateurs et ajustant sa performance selon leurs réactions ou, plus largement, selon l'ambiance du moment. Dans le cas d'une joute de slam, les spectateurs font partie de la performance, de ce «ici et maintenant », de cette immédiateté, c'est-à-dire qu'ils sont ancrés dans l'espace et le temps de la performance. L'immédiateté est au cœur de la notion de performance poétique, puisque c'est probablement l'élément qui la différencie le plus de la poésie écrite. Le message poétique est mis en scène, non comme un simple jeu théâtral, mais plutôt comme une quatrième dimension de la poésie, où une interaction a lieu entre le slameur, son texte et les spectateurs. Bref, l'auditeur (ou le spectateur) contribue précisément à la production de la performance: «Il est auditeur-auteur, à peine moins que n'est auteur exécutant. D'où la spécificité du phénomène de réception dans la poésie orale » (Zumthor, 1983 : 234). En d'autres mots, la performance « désigne un acte de communication comme tel; réfère donc à un moment saisi comme présent. Le mot signifie la présence concrète de participants impliqués dans cet acte de manière immédiate » (Zumthor, 1990 : 54-55).

La performance actualisée dans une joute de slam fait du slameur l'interprète de ses propres textes. Le contexte (scène ouverte, joute régulière, finale régionale, joute scolaire, par exemple) variera toutefois, en plus des spectateurs présents, de leurs perceptions sensorielles et de leurs réactions immédiates, car certains auront déjà un horizon d'écoute (Vorger, 2011), c'est-à-dire des attentes à l'égard du texte slamé (ils pourront, par exemple, l'avoir déjà entendu dans une autre joute), du slameur (thèmes qu'il privilégie d'une joute à l'autre ou d'une manche à l'autre, style de performance, attitude, âge, etc.) ou, plus largement, du slam (comme spectacle, art oratoire, poésie de performance, etc.). Par conséquent, les performeurs peuvent, au moment d'entrer sur scène selon l'ambiance générale de la joute ou même pendant leur performance de trois minutes, ajuster ou modifier leur voix, une mimique ou même quelques mots de leur texte selon les réactions des spectateurs, voire ajouter des silences de quelques secondes si les spectateurs rient trop fort, les empêchant d'être momentanément clairement entendus : les spectateurs sont manifestement partie prenante de la performance poétique. Partant, le performeur accepte que sa performance n'existe jamais en elle-même : l'interaction de 
tous les éléments de l'environnement peut présenter un apport comme un piège pour celui qui prend le risque de voir sa performance ou des passages de son texte transformés par la réception instantanée des spectateurs.

Aux dimensions texte, temps, espace et spectateurs s'ajoutent les dimensions spécifiques au performeur/slameur, soit le corps et la voix, ainsi que les finalités de sa performance. Trois niveaux de mouvement corporel se dégagent des performances : le geste (ex. : main sur le cœur, poing fermé), la mimique (ex. : yeux fermés, sourire, sourcils froncés) et la danse (posture statique ou dynamique) (Zumthor, 2008 : 187). Le geste « engendre dans l'espace la forme externe du poème. Il en fonde l'unité temporelle, en la scandant de ses récurrences $»$ (Zumthor, 1983 : 197). À contrario, le slameur peut aussi poser un geste zéro, c'est-à-dire qu'intentionnellement, il contrôlera ses gestes et mimiques pour créer une absence momentanée de mouvement corporel : "La vocalité poétique [...] parfois confère une fonction au silence : de même, la gestualité peut intégrer de manière significative des "gestes zéro" » (Zumthor, 1983: 197). Si les silences sont capables de parler dans la poésie écrite, ceux-ci étant illustrés notamment par des espaces ou des marques de ponctuation, ils le sont également dans la performance: l'esthétique créée par le silence intentionnel dans la mise en scène, Zumthor la décrit comme suit : «La mimique seule ou le seul regard (à l'exclusion de tout autre geste) se rencontre, exceptionnellement, en performance lorsque l'exécutant veut provoquer, dans le reste de l'espace corporel, un "effet zéro" » (1983 : 197). Par exemple, quand un slameur reste debout sur scène devant des spectateurs, même s'il ne parle pas, le langage du corps montre une intention (pour illustrer, par exemple, l'insistance, l'étonnement, la stupéfaction). Cela exige un niveau élevé d'attention des spectateurs pour réagir à cet « effet zéro » chargé d'un sens profond.

En outre, la dimension sonore de la poésie est incontournable, car le texte oral du slam, transmis directement par la voix, se distingue de "l'écriture à haute voix » (Barthes, 1973 : 104-105), de «l'orature » (Hagège, 1987 : 110) ou de «l'auditure » (Bobillot, 2009: 66-68), car il s'agit d'un texte qui existe pour être spécifiquement produit et reçu oralement. Cela engage une mise en scène de la voix qui relève du corps et de l'engagement de soi : " La voix n'est plus réductible au phonique car l'énergie qui la produit engage aussi le corps vivant avec son histoire. Le rythme est le mouvement de la voix dans l'écriture. Avec lui, on n'entend pas du son, mais du sujet» (Meschonnic, 1989 : 317). La voix est un «discours-en-présence, modulé par les rythmes du corps, imprégné de sensualité chaleureuse, brouillé de bruits quotidiens, peu nuancé mais immédiatement impératif dans la vérité de son évidence » (Zumthor, 1983 : 274). Le véritable défi de la performance poétique se trouve alors dans l'interprétation orale et physique du texte dont la finalité est de faire réagir les spectateurs, par le rire, l'émoi et l'empathie, notamment.

\section{Méthodologie}

Dans le cadre d'une recherche qui visait à comprendre ce qui caractérise les performances poétiques de slameurs, nous avons observé, filmé et analysé 104 performances réalisées par 31 différents slameurs québécois (Émery-Bruneau et Yobé, 2015). Ces données ont été collectées auprès d'une équipe officielle de la Ligue québécoise de slam (LIQS), SlamOutaouais (Gatineau). Nous avons analysé six joutes de slam qui se sont déroulées en sept mois. Nous avons choisi cette équipe nationale pour sa 
représentativité, plus particulièrement parce qu'elle est composée de slameurs âgés de 16 à 75 ans et d'origines diverses, ce qui offre un échantillon très diversifié de participants. De plus, puisque cette équipe fait partie de la LIQS, elle procède de la même façon et avec les mêmes règles que les autres équipes nationales de slam. En outre, depuis 2009, à trois reprises, les champions nationaux de la LIQS provenaient de cette équipe, ce qui ajoute à leur reconnaissance et représentativité du milieu slam.

Les compétitions régionales ont mené à une finale régionale. Ainsi, lors des joutes régulières, le gagnant se voyait attribuer une place en demi-finale. À la suite des deux demi-finales, les huit slameurs qui ont obtenu les points les plus élevés lors de ces joutes ont non seulement été considérés comme les meilleurs slameurs de la saison, mais ont été retenus pour participer à la finale régionale (quatre hommes; quatre femmes) au cours de laquelle ils ont tous dû présenter deux slams originaux. Pour cet article, nous avons décidé de travailler sur un échantillon réduit, mais représentatif de notre échantillon plus large, en nous concentrant spécifiquement sur les 16 performances poétiques de la joute finale au cours de laquelle les meilleurs slameurs ${ }^{4}$ régionaux ont été consacrés. En outre, la diversité des spectateurs était non négligeable étant donné que lors de la finale, le cafébar où se tenait la joute était rempli de spectateurs, jeunes et plus âgés, habitués à ces soirées et passionnés de slam, alors leurs réactions reflétaient leur réception à la fois singulière et collective, teintant par conséquent les performances poétiques que nous avons observées et analysées.

Nous avons procédé à une double analyse de nos données. D'abord, nous avons procédé à une analyse de contenu des textes afin de dégager les thèmes, les discours ainsi que les figures et procédés récurrents. Puis, nous avons croisé à cette première analyse une analyse visuelle et sonore des performances, en tenant compte des principales dimensions décrites dans le cadre théorique, soit celle de la voix, du corps et des réactions des spectateurs. Ces analyses nous ont amenées à dégager ce qui caractérise des performances poétiques produites lors d'une joute de slam au moment précis de la rencontre entre la performance du slameur et les réactions des spectateurs.

\section{Analyse et interprétation des données}

Nous avons identifié trois aspects émergents qui se dégagent de notre corpus et qui nous permettent d'expliquer les caractéristiques des performances poétiques observées : 1) l'antinomie, l'anaphore et la litanie comme procédés récurrents pour obtenir l'empathie; 2) la purge bilatérale entre la catharsis des slameurs et celle des spectateurs; 3) l'humour comme gage d'engagement des spectateurs.

\subsection{L'antinomie, l'anaphore et la litanie pour créer l'empathie.}

L'antinomie, récurrente dans les textes slamés, est une figure d'opposition ou de contradiction, réelle ou apparente, distinguant les actions de chacun en illustrant une

\footnotetext{
${ }^{4}$ Nous devons toutefois préciser que pour respecter le caractère confidentiel des renseignements fournis par les participants à la recherche, et ce, même si les joutes de slam étaient publiques, nous ne pouvons pas divulguer leur nom alors nous utilisons des initiales pour anonymiser nos données (ex. : F.H.), comme l'exige le Comité d'éthique à la recherche de l'Université du Québec en Outaouais. Puisque tous les slameurs performaient dans les deux manches obligatoires de la joute, nous avons donc ajouté un chiffre à la suite des initiales pour identifier la manche au cours de laquelle sa performance avait été réalisée (ex. : F.H.1 ou F.H.2).
} 
réaffirmation de la différence entre Je et l'Autre (soi / altérité), par exemple « Je t'ai vu naitre / Tu me verras mourir» (D.T.1) ou « Dans le froid de la nuit, ma tendre amie, il faudra se dépêcher de consommer cette chaleur» (T.B.1). Le recours à cette figure marque systématiquement une distance avec l'Autre, montrant du même coup des personnages confrontés à eux-mêmes dans leur rapport au monde. Dans ces récits de soi slamés, souvent autofictifs, les slameurs ne peuvent exister sans cet Autre qui est non seulement le destinataire réel venu les entendre et les voir (spectateurs), mais qui est aussi le personnage créé à partir de leurs expériences réelles. La division entre le Je et l'Autre est un procédé utilisé fréquemment pour illustrer cette dualité, par exemple «L'un est fidèle face à lui-même et l'autre a tort » (Y.B.2), «Et qu'est-ce que tu fais quand t'existes plus sur aucune map, dans aucun registre, sur aucune carte? » (A.A.2), « On vous ment, le gouvernement me garde en vie» (M.B.2), "Tu mourrais en couche, au nom de crucifix » (D.T.2). Ce procédé crée une dualité entre le monde réel du slameur (besoin de se raconter, de s'adresser à un destinataire réel - spectateurs qui voient, écoutent, ressentent, se remémorent une performance) et la fiction dans laquelle la rencontre entre les protagonistes semble absente.

Dans cette performance poétique, ces antinomies se transforment souvent en figure de litanie, c'est-à-dire qu'elles deviennent l'action d'un dialogue qui se compose d'une série de brèves invocations ou de supplications sacrées qu'une personne récite ou chante, tout en cherchant à engager d'autres personnes dans la prière répétée. Pour les slameurs, le but est de maintenir une cadence dans leur performance et un écho dans la mémoire des spectateurs qui, par la force du procédé, obligent ceux-ci à (ré)entendre cette litanie et à y trouver un repère dans le rythme et l'intertexte, ainsi que dans le corps. À titre d'exemple, la performance de D.T. $1^{5}$ est particulièrement éloquente. Slameuse âgée d'une soixantaine d'années, son slam, un récit de soi, raconte l'amour maternel ( $\mathrm{Je}$ ) pour un enfant (Léon). Pendant toute sa performance, la slameuse sourit et regarde les spectateurs. Ses mains sont ouvertes comme si elle priait, ses bras formant un V de ses coudes collés à ses flancs à ses doigts pointant vers le plafond/ciel. Elle maintient cette position pendant les trois minutes de son slam. S'adressant à Léon, dont le prénom est répété à 18 reprises pendant la performance, cette gestuelle illustre l'accueil qu'elle lui offre en tant que grand-mère : bras ouverts, elle est prête à le serrer dans ses bras pour lui exprimer toute l'affection qu'elle ressent pour lui, et lui procurer la protection maternelle qu'elle souhaite lui offrir. Les huit fois où elle répète « Je t'ai vu naitre / Tu me verras partir / Mais entre les deux Léon... », elle change de ton afin de marquer plus fortement l'intensité de cette apostrophe au destinataire. Ce changement de ton est porté par une litanie qui, pour elle, est ce lien qui les unit pour toujours: «Ensemble Léon nous créerons des moments de sublime et d'éternité ». Or, cette litanie de leur relation fait aussi ressortir la dualité entre le long futur d'un nouveau-né et le temps de vie potentiellement plus restreint d'une personne beaucoup plus âgée. Tout au long de la performance, sa voix est teintée de tendresse : c'est le ton réconfortant d'une figure maternelle s'adressant à son (petit)-enfant. Son slam est d'ailleurs marqué d'une intertextualité forte par les paroles d'une comptine pour enfant provenant de la très populaire série télévisuelle québécoise Passe-Partout: «J'ai deux yeux tant mieux / Deux oreilles, c'est pareil [...] Deux fesses qui se connaissent ». L'intertextualité

${ }^{5}$ Tirée de la vidéo $00018: 13 \mathrm{~m} 30-16 \mathrm{~m} 20$

Language and Literacy

Volume 18, Issue 1, 2016

Page 46 
est ainsi utilisée comme une clé pour entrer en communication avec « [s] on Léon » et à le protéger en priant des forces plus puissantes de prendre soin d'eux (position de prière : mains ouvertes et bras élevés). En plus de présenter une réalité antithétique (naissance d'un petit-fils, mort de la grand-mère) martelée d'une anaphore de l'altérité (Léon, n=18) et de convoquer la mémoire intertextuelle, la litanie joue un rôle très précis dans cette performance poétique: elle engage les spectateurs dans un mouvement commun d'empathie pour l'idéal de l'amour maternel inconditionnel, laquelle est portée par une comptine chantée à l'enfant et soutenue par des référents culturels populaires (intertextualité) pouvant rappeler aux spectateurs leur propre enfance. Pendant la performance, les spectateurs ont écouté attentivement cette litanie et certains présentaient même des mimiques (têtes penchées, sourcils relevés) illustrant l'empathie qu'ils éprouvaient pour le personnage.

Sur un autre ton, J.L. $2^{6}$ utilise aussi l'anaphore en répétant à 11 reprises « Je suis tannée » en parlant des relations hommes-femmes qu'elle considère superficielles, insatisfaisantes et frustrantes. Systématiquement, elle met le ton en disant «Je suis tannée », avec une voix forte et balançoire. Son slam marque une tension entre le besoin d'une femme d'être désirée pour ce qu'elle est et le désir de l'homme plutôt centré sur le paraitre :

\author{
Je suis tannée \\ Tannée des femmes plastiques \\ Pas compliquées, pleines de caprices \\ Que les femmes soient belles, fassent semblant \\ "Souris, je veux voir tes dents \\ Mais ne mords pas » [...] \\ Je suis tannée \\ Des hommes lisses \\ Hypocaloriques \\ Gym tonic \\ Power et Power trip \\ Les messieurs voyages tout inclus \\ Chromés, bien vêtus \\ Mais, c'est pas de ta faute mon beau \\ $C$ 'est ta cravate qui t'embête le complet \\ Qui t'empêche le cour de parler de la tête \\ Comme le reste, puis les autres \\ Copy-paste \\ Pareil au même \\ Je suis tannée...
}

Chaque fois que sa litanie est relancée, elle interjette un silence avant de (ré)affirmer « Je / suis / tannée » avec détermination et assurance : elle demeure debout et ferme ses yeux quand elle partage cette insatisfaction des relations fausses. Puis peu à peu, sa litanie de la superficialité se transforme en litanie de l'angoisse, marquée par

${ }^{6}$ Tiré de la vidéo $00022: 1 \mathrm{~m} 30-4 \mathrm{~m} 40$

Language and Literacy

Volume 18, Issue 1, 2016

Page 47 
l'anaphore de la peur: «Mais j'ai peur / Peur du reflet que me renvoie la société / Dans mon miroir que je n'ose plus affronter / Peur que de n'y voir qu'une surface / Un masque façonné pour plaire et se taire / Une façade ». L'expression de la crainte prend place dans la performance et, progressivement, le volume de sa voix diminue jusqu'à ce qu'elle admette au dernier vers « Je veux changer, et ça commence ce soir », suivi d'un silence avant les applaudissements des spectateurs : empathiques à son état douloureux, ils laissent paraitre des mimiques faciales témoignant de leur impuissance à la consoler. Or, ce ton est privilégié par cette slameuse, car le premier slam qu'elle a présenté dans cette joute engageait aussi, sinon davantage, les spectateurs à être empathiques, voire à participer à une « purgation des passions », comme nous l'expliquons plus bas.

\subsection{Catharsis : de la purgation des slameurs à celle des spectateurs.}

Dans sa Poétique, Aristote estimait que dans la tragédie grecque le spectacle et la musique étaient des moyens forts de l'émotion: il s'agit d'un des effets du spectacle autant que du texte. Cet effet, la catharsis, qui désigne les émotions des spectateurs à l'égard de l'art théâtral, peut s'étendre à d'autres genres, dont la comédie ou la poésie, car il s'agit d'une des finalités de la fiction littéraire, comme l'ont entre autres montré depuis une dizaine d'années les théoriciens de la lecture subjective (Langlade et Rouxel, 2004). Mais dans les joutes de slam, cette «purgation des passions » est double, la projection de soi traversant le quatrième mur dans les deux sens : d'un côté, le slameur partage ses émotions sur scène, de l'autre, les spectateurs projettent leurs émotions dans le poème ou le récit de soi slamé et y réagissent par le rire, la passion ou l'empathie, par exemple, ce qui s'observe dans leurs mimiques, leurs claquements de doigts, leurs éclats de rires, etc. Et c'est précisément au moment de cette rencontre qu'est créée la performance poétique, marquée d'une existence éphémère d'au plus trois minutes, règle de la joute. En fait, « la situation performantielle apparait donc comme une opération cognitive et je dirais même plus précisément fantasmatique. Elle est un acte performatif de celui qui regarde et de celui qui fait» (Zumthor, 1990: 45). Et nous l'observons notamment par cette purge bilatérale entre la catharsis des slameurs et celle des spectateurs, desquelles jaillit d'ailleurs souvent l'empathie.

L'exemple du slam de J.L. ${ }^{7}$ est éloquent en ce sens. Dès qu'elle arrive sur scène, elle fait une mimique affirmative avec son regard qui semble vouloir dire qu'elle va commencer et qu'elle n'a pas une minute à perdre. D'ailleurs, l'obsession du temps qui défile est exprimée dès son premier vers : "J'ai trois minutes pour te parler, mais la nostalgie me pogne ». Ce premier vers illustre la tension entre le temps qui avance, le regret mélancolique d'un amour perdu et le détachement, thèmes de ce slam. Pendant sa performance, elle donne le rythme de ses mots mémorisés avec sa main gauche, comme un métronome conscient du temps qui défile : « Mais la nostalgie m'arrête encore / Et j'ai déjà perdu trente d'entre elles [secondes] à me justifier ». Ce geste, ainsi que ses vers qui rappellent le compte à rebours auquel elle est confrontée ( «J'ai trois minutes pour te parler $[\ldots]$ trois minutes c'est peu, c'est rien, c'est pas grand-chose [...] je prendrai 180 secondes de ton temps [...] À peine 60 secondes [...] Nostalgie de 20 secondes [...] Mon impuissance devant ces dernières secondes ») est aussi une mise en abyme forçant les spectateurs à ressentir non seulement de l'empathie pour son processus de détachement

${ }^{7}$ Tiré de la vidéo $00019: 0 \mathrm{~m} 15-3 \mathrm{~m} 25$ 
(nostalgie d'un amour perdu), mais aussi l'angoisse de respecter les trois minutes règlementaires de la performance slamée dans le cadre de cette joute finale. Et cette purge commune est partagée à la fin de son slam lorsqu'elle dit « Je voulais juste y croire plus longtemps! », et que les spectateurs applaudissent vigoureusement alors que d'autres s'exclament «WOW! ». En plus de son obsession du temps qui avance et l'éloigne de son amour passé, elle se sent amère, voire frustrée de ne pas arriver à anticiper positivement le futur, frustration aussi exprimée avec son corps, car pendant toute la performance, elle tient sa robe d'une main ferme comme si elle cherche à se rattacher à quelque chose. Quand elle dit le mot nostalgie, répété 17 fois, elle ferme les yeux, pour exprimer son repli sur elle-même, mimant avec son visage la mélancolie qu'elle ressent. Mais la raison l'amène à être sarcastique : "Tu me manques, surtout quand tu es là / Et le temps me manque aussi ». Elle dit ce vers en séparant chaque mot d'un bref silence, marquant avec insistance son besoin d'être entendue par l'autre, ce qui la pousse à s'exprimer lentement, de façon pesée, rythmée et assurée. Ce ton ouvre la voie aux confidences et marque de nouveau une quête d'empathie, de l'Autre comme des spectateurs : "Lorsqu'hier semble si loin/Il faut se sentir devenir folle/Toujours se croire l'année dernière, l'été dernier/Nostalgie de perdre la carte ». Mais quand elle termine sa performance, la purge a finalement eu lieu : la performance achevée, elle remercie les spectateurs en souriant, puis retourne à sa condition de spectatrice de la joute. La distance et la douleur sont reflétées dans toute la performance, ce qui engage les spectateurs non seulement dans un mouvement d'empathie, mais aussi de purge, c'est-àdire d'une catharsis du sentiment d'être rejeté que plusieurs peuvent avoir vécu dans différentes circonstances (séparation amoureuse, amitié déchue, etc.). Elle vit un détachement et elle le crie avec sa voix à la fois forte et frêle, ce que plusieurs spectateurs s'identifiant à la slameuse auraient aussi peut-être souhaité faire lorsqu'ils ont vécu une telle souffrance à la suite d'un rejet.

Dans la performance poétique, le slameur trouve un espace d'expression de soi auquel s'identifie le spectateur: c'est la purgation ou la catharsis aristotélicienne (Aristote, 335 av. J.-C./1990 : 93). Cette catharsis est aussi vécue par les spectateurs, miroir des purgations exprimées sur scène, les projetant à leur tour sur le slameur qui se manifeste, perçoit, réagit et permet aux spectateurs de vivre à leur tour une purgation. Cette dialectique amène d'emblée les spectateurs à vivre à travers le point de vue de l'Autre. S'impliquer dans cette dialectique sans crainte de leurs effets réels peut aider à mieux se comprendre soi-même et à ne pas répéter la chaine de décisions de l'histoire racontée dans le texte du slameur :

«Dans la vie, nous faisons cela à chaque pas: nous nous apprécions nous-mêmes du point de vue des autres, nous essayons de comprendre les moments transgrédients à notre conscience même et d'en tenir compte à travers l'autre [...] en un mot: constamment et intensément, nous surveillons et saisissons les reflets de notre vie dans le plan de conscience des autres hommes [...] Je se cache dans l'autre et dans les autres, il ne veut être qu'un autre pour les autres, entrer jusqu'au bout dans le monde des autres en tant qu'autre, rejeter le poids du je unique au monde (le je-pour-soi). » (Todorov, 1981 : 145-150). 
Ce rapport dialectique s'actualise dans la performance, car « communiquer ne consiste pas à seulement faire passer une information; c'est tenter de changer celui à qui l'on s'adresse; recevoir une communication, c'est nécessairement subir une transformation » (Zumthor, 1990, 57). Mais alors, la catharsis serait-elle la finalité la plus éminente de la performance poétique, ou à tout le moins du slam?

\subsection{L'humour : gage d'engagement des spectateurs.}

Nous avons montré ailleurs que l'utilisation des néologismes de type mots-valises, très fréquents dans les textes des slameurs, contribue à provoquer des réactions fortes chez les spectateurs, lesquelles sont souvent exprimées par le rire (Émery-Bruneau et Yobé, 2015). Mais en plus des mots-valises, qu'est-ce qui pousse le plus les spectateurs à rire dans une performance poétique, comme nous l'avons observé, sinon l'humour? Car bien que l'humour s'adresse à l'esprit, le rire s'exprime par la voix et affecte par la même occasion le corps. Forme d'esprit, figure de rhétorique, procédé stylistique, etc., l'humour est toujours à définir, car indéfinissable (Grojnowki et Sarrasin, 1990). Mais nous pouvons néanmoins affirmer que l'humour est un procédé généralement utilisé comme mode d'expression résultant de cette attitude ou pour illustrer l'absurdité d'un contexte, par exemple en soulignant les préjugés ou les manques, ou alors les aspects plaisants ou insolites de la réalité. Par conséquent, l'humour est un procédé qui interpelle très souvent le grand public, car il participe au comique, il contribue à la promotion de l'absurde et il devient un élément rassembleur dans une communauté :

«La démarche humoristique vise la dislocation de la réalité apparente. L'Homme veut exorciser son angoisse en dégonflant les menaces de l'environnement. Il doit briser les automatismes confortables de la vie en société. Par l'humour, l'Homme se protège... et attaque. » (Dupuis, $1984: 17$ )

Mais comment l'humour existe-t-il dans les performances poétiques de slam? Parmi les performances de notre échantillon, au moins cinq des seize slams de cette finale régionale peuvent être qualifiés d'humoristiques, bien que ces cinq performances aient créé des effets différents selon les procédés utilisés (l'ironie, la parodie, l'imitation et le comique). Or, deux des trois slameurs qui se sont classés parmi les champions de cette finale régionale ont utilisé l'humour dans leur performance: ce genre provoquant généralement le rire et l'amusement chez les spectateurs, il semble aussi être un tremplin efficace vers une réception plus positive des spectateurs, ce qui permet par conséquent aux slameurs d'obtenir des points plus élevés octroyés par les cinq juges répartis parmi les spectateurs. Nous décrivons ici deux performances qui ont été particulièrement portées par l'humour et systématiquement accompagnées de rires des spectateurs : la première, de M.B.1, est plutôt ironique, alors que la seconde est une parodie (D.D.2). Ces performances humoristiques permettent aux slameurs d'entrer en interaction avec les spectateurs et de vivre un moment «colludique » (Vorger, 2015), notion renvoyant à colludere, soit «jouer ensemble», et à la réception puisque ce jeu implique inévitablement le slameur (action du locuteur) et l'auditeur dans son processus : le slam c'est l'art d'impliquer « l'auditor in poema » (Vorger, 2015). 
Avant même le cri d'encouragement «Parle!» lancé à l'unisson par les spectateurs pour ouvrir la performance, M.B. $1^{8}$ saute sur scène, démontrant un dynamisme et une excitation à prendre le micro pour le retirer de sa base, geste qui confirme qu'il va s'emparer de la scène avec son corps - et non seulement utiliser le micro avec sa voix. Dès le premier vers, il provoque les premiers rires des spectateurs en disant sarcastiquement « Eille! Je suis beau », puis après le troisième vers, il donne le ton en disant «Les jeunes, on a une mauvaise réputation. Juste hier, le père de mon ami, monsieur Jean, me dit: "Avec un jeune, c'est impossible d'avoir une véritable conversation. En plus, vous êtes violents." ». Son texte devient alors un dialogue entre un Je fier et affirmé (ce «jeune ») et une figure de l'altérité, un certain " Monsieur Jean » (représentant un père porteur de préjugés et de stéréotypes à l'égard des «jeunes »). Ce dialogue lors de la performance donne à penser à une conversation pouvant être tout à fait réaliste entre un spectateur et ce slameur aux cheveux orange. Au même titre que les mimiques utilisées pendant sa performance, le slameur adopte un ton oscillant entre la naïveté et l'ironie, comme dans cet extrait : " "Les jeunes n'apprendront jamais à gérer leur argent" / C'est faux, j'apprends! / La preuve, chaque fois qu'on m'offre de l'argent, je la prends / Et que celui qui n'a jamais mal géré son argent/me lance la première piasse ». De tels vers ironiques provoquent d'ailleurs systématiquement des éclats de rires dans la salle, car la performance vise aussi à faire réagir les spectateurs avec ce discours humoristique qui reprend un à un les stéréotypes et préjugés sur les jeunes à partir du point de vue du "père » (ex. : les jeunes sont violents, drogués, axés sur le paraitre - hommes musclés ou femmes mannequins, ne pensent qu'au sexe et qu'à consommer des biens futiles, agissent de façon irréfléchie, etc.), puis déconstruit chaque argument de ce discours par une réplique ironique visant à amener les spectateurs à partager le point de vue défendu par le « jeune ». L'ironie fait non seulement ressortir ce dialogue de sourds, mais force d'emblée les spectateurs à adhérer à la position du « jeune », ce slameur dynamique, fier, défendant avec ferveur sa génération contre un regard paternaliste, cliché et traditionnel. La performance se termine par des rires en chœur, de forts applaudissements et cris d'encouragement du public après que le slameur eut lancé son dernier vers ironique : «Bon, maintenant que j'ai tout dit / Je vais retourner dans mon taudis jouer à mes veux vidéos / En bon jeune que je suis ».

Le deuxième exemple de slam humoristique est une parodie qui renvoie à la caricature d'un personnage connu, mais dont on exagère les mimiques, les gestes, les tics stylistiques, etc., et où on systématise jusqu'à l'absurde les procédés littéraires, on exagère les opinions émises et, surtout, on vise la satire. L'exemple du slam de D.D. $2^{9}$ est justement la parodie d'un discours politique de Stephen Harper, Premier ministre du Canada, dans lequel se trouve une abondance de mots-valises sur lesquels la sémantique politique est transposée en sémantique de l'abus sexuel. Dans ce slam, la position de son corps est adaptée au discours qu'il parodie : ses mains prennent part à la performance et essaient d'attirer les spectateurs en faisant un mouvement avant-arrière avec le bras, pour les engager avec lui. De plus, le slameur a adapté sa voix de façon à s'exprimer avec un accent anglais marqué, proche de celui qu'il parodie. Malgré les éclats de rire très forts après chaque mot-valise et chaque vers, le slameur maintient une attitude sérieuse,

\footnotetext{
${ }^{8}$ Tiré de la vidéo $00018: 17 \mathrm{~m} 50-20 \mathrm{~m} 30$
}

${ }^{9}$ Tiré de la vidéo $00021: 3 \mathrm{~m} 00-5 \mathrm{~m} 50$ 
comme s'il produisait un discours politique respectable et comme si les spectateurs étaient réellement ce peuple canadien écoutant le Premier ministre, ce qui crée un puissant effet à la performance parce que le slameur incarne vraiment le personnage qu'il parodie, exagérant néanmoins les mouvements du corps pour mieux faire ressortir l'absurdité du contrôle de soi d'un homme politique. Contrairement à d'autres performances dans lesquelles le langage montre une expérience personnelle de la passion ou de la douleur de façon parfois plus ou moins évidente, dans le cas de cette parodie, c'est le sens de ses mots qui est essentiel à une réception vivement réactive : une personne qui n'aurait pas les référents culturels ne pourrait pas comprendre pourquoi les spectateurs rient ainsi à s'époumoner, pouvant même être outrés par la sémantique sexuelle de ce texte. Les spectateurs ont donc une complicité avec le slameur et sont dès lors partie prenante de cette performance poétique. Comme l'illustre l'extrait suivant, l'effet comique est provoqué par un contexte éminemment local, renvoie à l'évocation critique de références connues et utilise une sémantique du sexe pour tourner en ridicule un personnage notoire :

Mes très chers circoncitoyens canadiens

Je n'ai pas besoin de m'introduire dans vous

Depuis mes dernières érections

J'ai toujours donné une place ferme aux femmes

dans mon administration pubique

tout dans le but de fourrer les futures de demain

Notre regroupement politique est pour vous et porno [pour nous]

Nous redonnons beaucoup à la collectivité, ainsi qu'aux partis privés

En tant que Premier pénis

Étant moi-même génital de deux enfants

Je vous promets de protéger la classe moyenne et les bijoux de famille

J'espère pouvoir toucher le plus de Canadiens possible

Heureusement que le parti conservateur a beaucoup d'entrejambe

Nous sommes le plus grand parti intime du parlement [...]

Et j'aimerais terminer ce discours avec un peu de latin

Abeas partum bubble gum rectum

Votez P.C.! Et vive le Canada des États-Unis!

Ce discours politique dénonçant avec humour un viol sociopolitique a valu au slameur la première place à la finale régionale (Gatineau) et à la finale nationale (Montréal), ce qui l'a mené à la Coupe du monde de slam. Comme quoi les histoires drôles, voire les parodies à sens politique apparaissent non seulement comme une forme privilégiée de contestation, mais aussi comme un genre rassembleur et confirmant une communauté d'idées (Regamey, 2001: 44). Or, avec la parodie, la réception est fondamentale parce qu'elle implique toujours une compétence interprétative des spectateurs : si ces derniers ne saisissent pas, par exemple, les référents culturels, les modèles détournés de leurs sens initiaux ou les stéréotypes, le statut parodique de la performance peut être parfois complètement ignoré. D'ailleurs, «le rire, l'humour, le comique, quoique phénomènes universels, s'élaborent à partir d'un cadre événementiel, 
d'un appareil de croyances et de convictions dictées par des coordonnées spatiotemporelles spécifiques » (Horowitz et Menache, 1994 : 15).

\section{Que peut-on retenir des performances poétiques de slam?}

La joute de slam est composée de plusieurs performances poétiques. Pour qu'il y ait performance poétique, il ne doit pas qu'y avoir un texte original d'un slameur et sa performance « individuelle» vocale et corporelle; il doit également y avoir des spectateurs présents réagissant dans le même espace, pendant un temps et un moment immédiat et éphémère - avec leurs émotions, leurs voix et leurs corps. C'est l'interaction de l'ensemble de ces dimensions qui crée la performance poétique, car l'action de chacun en fait partie: bref, ce qui s'opère est plus valorisé que ce qui s'accomplit. La performance poétique est un jeu performatif de l'ensemble des acteurs présents (slameurs, spectateurs, slammestre, chronomestre, technicien du son). Ainsi, en plus des slameurs sur scène et des spectateurs, le technicien du son qui fait jouer une musique festive ou ludique à chaque entrée et sortie de scène des slameurs fait aussi partie de la performance poétique en teintant l'ambiance générale et l'espace sonore avant et après les trois minutes au micro des slameurs. De même pour le slammestre, soit l'animateur de la joute qui appelle le slameur à prendre le micro et qui rappelle les règles de la joute, dont celle de ne pas slamer plus de trois minutes (chronomestre à l'affut du respect de cette règle) à défaut de perdre des points : cet animateur s'adressant systématiquement aux spectateurs ajoute aussi à l'ambiance dans laquelle se réalisera la performance.

Par ailleurs, en ce qui concerne la voix des slameurs, nous avons observé qu'elle est souvent modulée, soit par un crescendo pour marquer la colère, la frustration ou la révolte, soit d'un decrescendo pour illustrer la peur, l'angoisse, la tristesse. L'intonation est non seulement adaptée aux émotions exprimées, mais aussi ajustée selon le personnage représenté (ex. : voix forte et dominante du père dans le slam de M.B.1 ou accent anglais prononcé dans le slam de D.D.2). Du côté des spectateurs, leurs voix se font parfois entendre pendant la performance, surtout par le rire, et à la fin, au moment des applaudissements qu'ils complètent de cris d'encouragements. Quant au corps, dans l'ensemble des performances, nous avons constaté que la "danse » au sens de Zumthor est plutôt statique, les slameurs demeurant devant le micro accroché à une perche ou, s'ils tiennent le micro dans leur main, ils se déplacent minimalement. Il en va de même des spectateurs, assis sur des chaises ou appuyés sur des tables de bistro. Pour ce qui est des gestes, les slameurs les utilisent à l'occasion pour illustrer une composante de leur texte (ex. : main qui bat le rythme du temps qui défile comme dans la performance de J.L.1 ou bras en V, pointant vers le haut, pour illustrer la litanie comme dans celle de D.T.1). Pour leur part, les spectateurs produisent régulièrement deux gestes fondamentaux : claquer des doigts pendant un slam pour exprimer leur intérêt pour un vers ou une mimique, entre autres, et applaudir à la fin d'une performance. Enfin, les mimiques faciales sont très présentes : les slameurs les utilisent pour mieux interpréter leur texte en illustrant, à l'aide de leur bouche, de leurs yeux ou de leurs sourcils, la tristesse, l'ironie ou la colère, entre autres. Du côté des spectateurs, ces mimiques sont aussi observées, mais semblent plutôt illustrer l'empathie qu'ils ressentent pour le slameur ou l'amusement d'une performance humoristique : leurs mimiques illustrent une part de leur réception spontanée. Ainsi, en tant qu'acte de langage, la performance poétique est-elle un modèle qui comprend au moins deux postures, situées dans un même espace et un même temps : une posture de la 
production (le slameur) et une posture de la réception (les spectateurs). Et pour saisir finement la performance poétique, l'analyse de l'interaction entre ces postures est fondamentale.

\section{Conclusion}

Poésie circonstancielle et populaire, le slam offre un espace d'expression à des slameurs (dont très peu, voire aucun, ne proviennent du milieu littéraire ou n'ont réalisé des études littéraires universitaires) voulant partager des textes qu'ils ont écrits et qu'ils performent devant des spectateurs provenant d'une variété de milieux sociaux, intéressés par le slam, perceptifs et réactifs pendant les performances. Mais en plus des réactions spontanées et immédiates des spectateurs, leur mémoire est une autre dimension, bien que plus difficilement observable, qui marque les performances poétiques et qui va au-delà des sens, puisqu'elle est la seule dimension qui permet la prévalence du texte slamé : « Il n'y a pas de performance sans action mémorielle, et celle-ci, perceptible à un seul ou à plusieurs niveaux de formalisation, peut être automatisée et intégrée au système ou, dans tel cas particulier, intentionnelle " (Zumthor, 1983: 224). La seule possibilité de perpétuité des performances est donc soustraite à la mémoire des spectateurs et à celle des slameurs : contrairement aux textes écrits et publiés, les performances poétiques, actes uniques et éphémères, ne perdureront que dans la mémoire collective ainsi que les mémoires singulières de ces «sujets-spectateurs ${ }^{10}$ », coauteurs de la singularité de ces performances par leur appréciation subjective et réflexive marquée de perceptions, d'émotions, de réactions et de réflexions, soit les dimensions les plus personnelles de leur réception. Mais comment pourrait-on saisir ce que les spectateurs et les slameurs retiennent des performances dans lesquelles ils ont été engagés, ce qu'ils vivent à rebours comme expérience, voire ce qui les a transformés dans leur manière de vivre la performance poétique? Dans cet article, nous avons pu dégager quelques caractéristiques générales de leur réception à l'aide d'éléments observables (mimiques, claquements de doigts, applaudissement, cris, rires), mais pour comprendre l'expérience vécue dans chaque performance poétique, il nous faudrait saisir ce que les slameurs et les spectateurs perçoivent, ressentent et ce dont ils prennent conscience pendant la performance, mais aussi après : que retiennent-ils, oublient-ils ou transforment-ils de cette performance éphémère à laquelle ils retournent de façon parcellaire dans leur mémoire? Voilà quelques questions sur lesquelles nous souhaitons désormais travailler pour mieux comprendre les effets de la performance poétique.

\section{Références}

Aristote. (335 av. J.-C./1990). Poétique. Paris : Le livre de poche.

Barthes, R. (1973). Le plaisir du texte. Paris : Seuil.

Bobillot, J.P. (2009). Poésie sonore, Éléments de typologie historique. Reims : Éditions Le clou dans le fer.

Brown, R. (2011). Promoting Cooperation and Respect. "Bad" Poetry Slam in the Nontraditional Classroom. Pedagogy : Critiqual Approaches to Teaching literature, Language, Composition, and Culture, 11(3), 571-578.

${ }^{10}$ Expression en écho à celle de « sujet lecteur » (Langlade et Rouxel, 2004). 
Chetouani, L. (2011). Le slam, poésie contemporaine, à l'école et au collège. Dans D. Banks (dir.), Aspects linguistiques du texte poétique. Paris : L'Harmattan, 75-93. Dupuis, G. (1984). « De l'humour et de la poésie ». Urgences, 12, 7-18.

Émery-Bruneau, J. et V. Yobé (2015). La joute de slam : description de la pratique sociale et analyse de sa transposition didactique. Dans L. Lafontaine et J. Pharand (dir.), Littératie : vers une maîtrise des compétences dans divers environnements (p. 185-206). Montréal : Presses de l'Université du Québec.

Grojnowki, D. et B. Sarrasin (1990). L'Esprit fumiste et les Rires fin de siècle. Anthologie. Paris : José Corti.

Hagège, C. (1987). L'Homme de parole. Contribution linguistique aux sciences humaines, Paris : Fayard.

Horowitz, J. et S. Menache (1994). L'humour en chaire. Le rire dans l'Église médiévale. Paris : Labor et Fides.

Langlade, G. et Rouxel, A. (2004). Le sujet lecteur. Lecture subjective et enseignement de la littérature. Rennes : Presses universitaires de Rennes.

Low, B.E. (2001). Spoken Word: Exploring the Language and Poetics of the Hip Hop Popular. PHD Thesis, York University.

Low, B. E. (2011). Slam school : learning through conflict in the hip-hop and spoken word classroom. Stanford: Standford University Press.

Meschonnic, H. (1989). La rime et la vie. Paris : Verdier.

Regamey, A. (2001). " "Prolétaires de tous pays, excusez-moi!", Histoires drôles et contestation de l'ordre politique en ex-URSS », Hermès, 29, 43-52.

Todorov, T. (1981). Mikhaïl Bakhtine : le principe dialogique, Paris : Seuil.

Vorger, C. (2011). Poétique du slam : de la scène à l'école. Néologie, néostyles et créativité lexicale. Thèse de doctorat inédite, Université de Grenoble.

Vorger, C. (2015). Le slam ou l'écriture partagée : de l'atelier à la toile en passant par les manuels scolaires. Dans M.-F. Bishop et A. Belhadjin (dir.), Les patrimoines littéraires à l'école : tensions et débats actuels (p. 371-386). Paris : Champion.

Zumthor, P. (1983). Introduction à la poésie orale. Paris : Seuil.

Zumthor, P. (1987). La lettre et la voix de la littérature médiévale. Paris : Seuil.

Zumthor, P. (1990). Performance, réception, lecture. Longueuil : Le Préambule.

Zumthor, P. (2008). Oralité. Intermédialités : histoire et théorie des arts, des lettres et des techniques, 12, 169-202.

Biographies des auteurs

Judith Émery-Bruneau est professeure à l'Université du Québec en Outaouais (Gatineau). Ses domaines de recherche s'inscrivent dans le champ de la didactique du français et portent sur l'enseignement et l'apprentissage de la littérature, notamment les pratiques d'enseignement de la littérature au secondaire, la didactisation du slam et le rapport à la lecture littéraire d'étudiants en formation initiale à l'enseignement. Elle fait partie de deux regroupements de chercheurs: l'Équipe de Recherche Littératie et Inclusion (ÉRLI) et le Centre de recherche interuniversitaire sur la formation et la profession enseignante (CRIFPE). 
Nydia Pando, étudiante, termine sa Licence en lettres hispaniques à l'Universidad de Guadalajara, Jalisco, México. Elle a fait deux stages d'introduction à la recherche avec Judith Émery-Bruneau grâce à deux bourses, la première offerte par Globalink Mitacs et la seconde offerte par ERLI. Elle vient de publier son premier roman (2015) aux éditions Paraíso Perdido dont le titre est Mis cortos regresos. 